\title{
Estimation of Population Mean Using Known Correlation Coefficient And Median
}

\author{
J. Subramani \\ Department of Statistics \\ Ramanujan School of Mathematical Sciences \\ Pondicherry University \\ R V Nagar, Kalapet, Puducherry - 605014, India \\ Email: drjsubramani@yahoo.co.in \\ G. Kumarapandiyan \\ Department of Statistics \\ Ramanujan School of Mathematical Sciences \\ Pondicherry University \\ R V Nagar, Kalapet, Puducherry - 605014, India \\ Email: kumarstat88@gmail.com \\ Received 25 January 2013 \\ Accepted 2 February 2014
}

\begin{abstract}
The present paper deals with the two modified ratio estimators for estimation of population mean of the study variable using the linear combination of the known population values of the Correlation Coefficient and the Median of the auxiliary variable. The biases and the mean squared errors of the proposed estimators are derived and are compared with that of existing modified ratio estimators for certain natural populations. Further we have also derived the conditions for which the proposed estimators perform better than the existing modified ratio estimators. From the empirical study it is also observed that the proposed modified ratio estimators perform better than the existing modified ratio estimators.
\end{abstract}

Keywords: Bias, Class, Mean squared error, Natural populations, Simple random sampling

\section{Introduction}

The simplest estimator of population mean is the sample mean obtained by using simple random sampling without replacement (SRSWOR), when there is no additional information on the auxiliary variable available. Sometimes in sample surveys, along with the study variable $Y$, information on auxiliary variable $X$, correlated with $Y$, is also collected. This information on auxiliary variable $X$ may be utilized to obtain a more efficient estimator of the population mean. Ratio method of estimation is an attempt in this direction. This method of estimation may be used when (i) $X$ represents the same character as $Y$, but measured at some previous date when a complete count of the population was made and (ii) the character $X$ is cheaply, quickly and easily available. Consider a finite population $\mathrm{U}=\left\{\mathrm{U}_{1}, \mathrm{U}_{2}, \ldots, \mathrm{U}_{\mathrm{N}}\right\}$ of $\mathrm{N}$ distinct and identifiable units. Let $\mathrm{Y}$ is a study variable with value $Y_{i}$ measured on $U_{i} ; i=1,2,3, \ldots, N$ giving a vector $\mathrm{Y}=\left\{\mathrm{Y}_{1}, \mathrm{Y}_{2}, \ldots, \mathrm{Y}_{\mathrm{N}}\right\}$ and let $X$ is an auxiliary variable which is readily available. The problem is to estimate the 
population mean $\bar{Y}=1 / N \sum_{i=1}^{N} Y_{i}$ with some desirable properties on the basis of a random sample selected from the population $U$ using auxiliary information. When population parameters of the auxiliary variable $X$ such as Population Mean, Coefficient of Variation, Coefficient of Kurtosis, Coefficient of Skewness, Correlation Coefficient, Median are known, a number of estimators such as ratio, product and linear regression estimators and their modifications are proposed in the literature. Before discussing further about the modified ratio estimators and the proposed modified ratio estimators the notations to be used in this paper are described below:

- N - Population size

- $\mathrm{n}$ - Sample size

- $\mathrm{f}=\mathrm{n} / \mathrm{N}$ - Sampling fraction

- $\mathrm{Y}$ - Study variable

- $\mathrm{X}$ - Auxiliary variable

- $\overline{\mathrm{Y}}, \overline{\mathrm{X}}$ - Population means

- $\bar{y}, \bar{x}$ - Sample means

- $\mathrm{S}_{\mathrm{y}}, \mathrm{S}_{\mathrm{x}}$ - Population standard deviations

- $\mathrm{C}_{\mathrm{y}}, \mathrm{C}_{\mathrm{x}}-$ Co-efficient of variations

- $\rho$-Correlation Coefficient

- $\beta_{1}=\frac{N \sum_{i=1}^{N}\left(X_{i}-\bar{X}\right)^{3}}{(\mathrm{~N}-1)(\mathrm{N}-2) \mathrm{S}^{3}}$ - Coefficient of skewness of the auxiliary variable

- $\beta_{2}=\frac{\mathrm{N}(\mathrm{N}+1) \sum_{\mathrm{i}=1}^{\mathrm{N}}\left(\mathrm{X}_{\mathrm{i}}-\overline{\mathrm{X}}\right)^{4}}{(\mathrm{~N}-1)(\mathrm{N}-2)(\mathrm{N}-3) \mathrm{S}^{4}}-\frac{3(\mathrm{~N}-1)^{2}}{(\mathrm{~N}-2)(\mathrm{N}-3)}$ - Coefficient of kurtosis of the auxiliary variable

- $\mathrm{M}_{\mathrm{d}}-$ Median of the auxiliary variable

- $\mathrm{B}($.$) - Bias of the estimator$

- $\operatorname{MSE}($.$) - Mean squared error of the estimator$

- $\hat{\bar{Y}}_{\mathrm{i}}\left(\hat{\bar{Y}}_{\mathrm{p}_{\mathrm{i}}}\right)$ - Existing (proposed) modified ratio estimator of $\overline{\mathrm{Y}}$

The ratio estimator for estimating the population mean $\bar{Y}$ of the study variable $\mathrm{Y}$ is defined as

$\hat{\bar{Y}}_{\mathrm{R}}=\frac{\overline{\mathrm{y}}}{\overline{\mathrm{X}}} \overline{\mathrm{X}}=\hat{\mathrm{R}} \overline{\mathrm{X}}$

where $\hat{R}=\frac{\bar{y}}{\bar{x}}=\frac{y}{x}$ is the estimate of $R=\frac{\bar{Y}}{\bar{X}}=\frac{Y}{X}$

The ratio estimator given in (1) is more precise than the SRSWOR sample mean, when there exists a positive correlation between $\mathrm{X}$ and $\mathrm{Y}$. Further improvements are also achieved on the classical ratio estimator by introducing a large number of modified ratio estimators with the use of known parameters like, Coefficient of Variation, Coefficient of Kurtosis, Coefficient of Skewness and Population Correlation Coefficient, Median. The lists of modified ratio estimators together with their biases, mean squared errors and constants available in the literature are classified into two classes namely Class 1, Class 2 and are given respectively in Table 1 and Table 2 respectively. 
Table 1: Existing modified ratio estimators (Class 1) with the constants, the biases and the mean squared errors

\begin{tabular}{|c|c|c|c|}
\hline Estimator & Constant $\theta_{i}$ & Bias - $\mathrm{B}()$. & Mean squared error MSE(.) \\
\hline $\begin{array}{l}\hat{\overline{\mathrm{Y}}}_{1}=\overline{\mathrm{y}}\left[\frac{\mathrm{C}_{\mathrm{x}} \overline{\mathrm{X}}+\beta_{2}}{\mathrm{C}_{\mathrm{x}} \overline{\mathrm{x}}+\beta_{2}}\right] \\
\text { Upadhyaya and Singh }^{1}\end{array}$ & $\theta_{1}=\frac{C_{x} \bar{X}}{C_{x} \bar{X}+\beta_{2}}$ & $\frac{(1-f)}{n} \bar{Y}\left(\theta_{1}^{2} C_{x}^{2}-\rho \theta_{1} C_{x} C_{y}\right)$ & $\frac{(1-f)}{n} \bar{Y}^{2}\left(C_{y}^{2}+\theta_{1}^{2} C_{x}^{2}-2 \rho \theta_{1} C_{x} C_{y}\right)$ \\
\hline $\begin{array}{l}\hat{\overline{\mathrm{Y}}}_{2}=\overline{\mathrm{y}}\left[\frac{\beta_{2} \overline{\mathrm{X}}+\mathrm{C}_{\mathrm{x}}}{\beta_{2} \overline{\mathrm{x}}+\mathrm{C}_{\mathrm{x}}}\right] \\
\text { Upadhyaya and Singh }\end{array}$ & $\theta_{2}=\frac{\beta_{2} \bar{X}}{\beta_{2} \bar{X}+C_{x}}$ & $\frac{(1-f)}{n} \bar{Y}\left(\theta_{2}^{2} C_{x}^{2}-\rho \theta_{2} C_{x} C_{y}\right)$ & $\frac{(1-f)}{n} \bar{Y}^{2}\left(C_{y}^{2}+\theta_{2}^{2} C_{x}^{2}-2 \rho \theta_{2} C_{x} C_{y}\right)$ \\
\hline $\begin{array}{l}\hat{\bar{Y}}_{3}=\bar{y}\left[\frac{\beta_{1} \bar{X}+S_{x}}{\beta_{1} \bar{x}+S_{x}}\right. \\
\text { Singh }^{2}\end{array}$ & $\theta_{3}=\frac{\beta_{1} \bar{X}}{\beta_{1} \bar{X}+S_{x}}$ & $\frac{(1-f)}{n} \bar{Y}\left(\theta_{3}^{2} C_{x}^{2}-\rho \theta_{3} C_{x} C_{y}\right)$ & $\frac{(1-f)}{n} \bar{Y}^{2}\left(C_{y}^{2}+\theta_{3}^{2} C_{x}^{2}-2 \rho \theta_{3} C_{x} C_{y}\right)$ \\
\hline $\begin{array}{l}\hat{\overline{\mathrm{Y}}}_{4}=\overline{\mathrm{y}}\left[\frac{\beta_{2} \overline{\mathrm{X}}+\mathrm{S}_{\mathrm{x}}}{\beta_{2} \overline{\mathrm{x}}+\mathrm{S}_{\mathrm{x}}}\right. \\
\text { Singh }^{2}\end{array}$ & $\theta_{4}=\frac{\beta_{2} \bar{X}}{\beta_{2} \bar{X}+S_{x}}$ & $\frac{(1-f)}{n} \bar{Y}\left(\theta_{4}^{2} C_{x}^{2}-\rho \theta_{4} C_{x} C_{y}\right)$ & $\frac{(1-f)}{n} \bar{Y}^{2}\left(C_{y}^{2}+\theta_{4}^{2} C_{x}^{2}-2 \rho \theta_{4} C_{x} C_{y}\right)$ \\
\hline $\begin{array}{l}\hat{\bar{Y}}_{5}=\bar{y}\left[\frac{\beta_{2} \overline{\mathrm{X}}+\beta_{1}}{\beta_{2} \overline{\mathrm{x}}+\beta_{1}}\right. \\
\text { Yan and } \operatorname{Tian}^{3}\end{array}$ & $\theta_{5}=\frac{\beta_{2} \bar{X}}{\beta_{2} \bar{X}+\beta_{1}}$ & $\frac{(1-f)}{n} \bar{Y}\left(\theta_{5}^{2} C_{x}^{2}-\rho \theta_{5} C_{x} C_{y}\right)$ & $\frac{(1-f)}{n} \bar{Y}^{2}\left(C_{y}^{2}+\theta_{5}^{2} C_{x}^{2}-2 \rho \theta_{5} C_{x} C_{y}\right)$ \\
\hline $\begin{array}{l}\hat{\overline{\mathrm{Y}}}_{6}=\overline{\mathrm{y}}\left[\frac{\beta_{1} \overline{\mathrm{X}}+\beta_{2}}{\beta_{1} \overline{\mathrm{x}}+\beta_{2}}\right] \\
\text { Yan and Tian }{ }^{3}\end{array}$ & $\theta_{6}=\frac{\beta_{1} \bar{X}}{\beta_{1} \bar{X}+\beta_{2}}$ & $\frac{(1-f)}{n} \bar{Y}\left(\theta_{6}^{2} C_{x}^{2}-\rho \theta_{6} C_{x} C_{y}\right)$ & $\frac{(1-f)}{n} \bar{Y}^{2}\left(C_{y}^{2}+\theta_{6}^{2} C_{x}^{2}-2 \rho \theta_{6} C_{x} C_{y}\right)$ \\
\hline $\begin{array}{l}\hat{\bar{Y}}_{7}=\bar{y}\left[\frac{C_{x} \bar{X}+\beta_{1}}{C_{x} \bar{x}+\beta_{1}}\right. \\
\text { Yan and Tian }{ }^{3}\end{array}$ & $\theta_{7}=\frac{C_{x} \bar{X}}{C_{x} \bar{X}+\beta_{1}}$ & $\frac{(1-f)}{n} \bar{Y}\left(\theta_{7}^{2} C_{x}^{2}-\rho \theta_{7} C_{x} C_{y}\right)$ & $\frac{(1-f)}{n} \bar{Y}^{2}\left(C_{y}^{2}+\theta_{7}^{2} C_{x}^{2}-2 \rho \theta_{7} C_{x} C_{y}\right)$ \\
\hline
\end{tabular}

Table 2: Existing modified ratio estimators (Class 2) with the constants, the biases and the mean squared errors

\begin{tabular}{|c|c|c|c|}
\hline Estimator & Constant $R_{i}$ & Bias - $\mathrm{B}()$. & Mean squared error MSE(.) \\
\hline $\begin{array}{l}\hat{\overline{\mathrm{Y}}}_{8}=\frac{\overline{\mathrm{y}}+\mathrm{b}(\overline{\mathrm{X}}-\overline{\mathrm{x}})}{\left(\beta_{2} \overline{\mathrm{x}}+\mathrm{C}_{\mathrm{x}}\right)}\left(\beta_{2} \overline{\mathrm{X}}+\mathrm{C}_{\mathrm{x}}\right) \\
\text { Kadilar and Cingi }{ }^{4}\end{array}$ & $\mathrm{R}_{8}=\frac{\beta_{2} \overline{\mathrm{Y}}}{\beta_{2} \overline{\mathrm{X}}+\mathrm{C}_{\mathrm{x}}}$ & $\frac{(1-f)}{n} \frac{S_{x}^{2}}{\bar{Y}} R_{8}^{2}$ & $\frac{(1-f)}{n}\left(R_{8}^{2} S_{x}^{2}+S_{y}^{2}\left(1-\rho^{2}\right)\right)$ \\
\hline $\begin{array}{l}\hat{\overline{\mathrm{Y}}}_{9}=\frac{\overline{\mathrm{y}}+\mathrm{b}(\overline{\mathrm{X}}-\overline{\mathrm{x}})}{\left(\mathrm{C}_{\mathrm{x}} \overline{\mathrm{x}}+\beta_{2}\right)}\left(\mathrm{C}_{\mathrm{x}} \overline{\mathrm{X}}+\beta_{2}\right) \\
\text { Kadilar and Cingi }{ }^{4}\end{array}$ & $\mathrm{R}_{9}=\frac{\mathrm{C}_{\mathrm{X}} \overline{\mathrm{Y}}}{\mathrm{C}_{\mathrm{x}} \overline{\mathrm{X}}+\beta_{2}}$ & $\frac{(1-f)}{n} \frac{S_{x}^{2}}{\bar{Y}} R_{9}^{2}$ & $\frac{(1-f)}{n}\left(R_{9}^{2} S_{x}^{2}+S_{y}^{2}\left(1-\rho^{2}\right)\right)$ \\
\hline $\begin{array}{l}\hat{\bar{Y}}_{10}=\frac{\bar{y}+b(\bar{X}-\bar{x})}{\left(\beta_{1} \bar{x}+\beta_{2}\right)}\left(\beta_{1} \bar{X}+\beta_{2}\right) \\
\text { Yan and Tian }{ }^{3}\end{array}$ & $R_{10}=\frac{\beta_{1} \bar{Y}}{\beta_{1} \bar{X}+\beta_{2}}$ & $\frac{(1-\mathrm{f})}{\mathrm{n}} \frac{\mathrm{S}_{\mathrm{x}}^{2}}{\overline{\mathrm{Y}}} \mathrm{R}_{10}^{2}$ & $\frac{(1-f)}{n}\left(R_{10}^{2} S_{x}^{2}+S_{y}^{2}\left(1-\rho^{2}\right)\right)$ \\
\hline $\begin{array}{l}\hat{\bar{Y}}_{11}=\frac{\bar{y}+b(\bar{X}-\bar{x})}{\left(C_{x} \bar{x}+\rho\right)}\left(C_{x} \bar{X}+\rho\right) \\
\text { Kadilar and Cingi }{ }^{5}\end{array}$ & $R_{11}=\frac{C_{X} \bar{Y}}{C_{x} \bar{X}+\rho}$ & $\frac{(1-f)}{n} \frac{S_{x}^{2}}{\bar{Y}} R_{11}^{2}$ & $\frac{(1-f)}{n}\left(R_{11}^{2} S_{x}^{2}+S_{y}^{2}\left(1-\rho^{2}\right)\right)$ \\
\hline $\begin{array}{l}\hat{\overline{\mathrm{Y}}}_{12}=\frac{\overline{\mathrm{y}}+\mathrm{b}(\overline{\mathrm{X}}-\overline{\mathrm{x}})}{\left(\rho \overline{\mathrm{x}}+\mathrm{C}_{\mathrm{x}}\right)}\left(\rho \overline{\mathrm{X}}+\mathrm{C}_{\mathrm{x}}\right) \\
\text { Kadilar and Cingi }{ }^{5}\end{array}$ & $\mathrm{R}_{12}=\frac{\rho \overline{\mathrm{Y}}}{\rho \overline{\mathrm{X}}+\mathrm{C}_{\mathrm{x}}}$ & $\frac{(1-f)}{n} \frac{S_{x}^{2}}{\bar{Y}} R_{12}^{2}$ & $\frac{(1-f)}{n}\left(R_{12}^{2} S_{x}^{2}+S_{y}^{2}\left(1-\rho^{2}\right)\right)$ \\
\hline $\begin{array}{l}\hat{\bar{Y}}_{13}=\frac{\bar{y}+b(\bar{X}-\bar{x})}{\left(\beta_{2} \bar{x}+\rho\right)}\left(\beta_{2} \bar{X}+\rho\right) \\
\text { Kadilar and Cingi }{ }^{5}\end{array}$ & $\mathrm{R}_{13}=\frac{\beta_{2} \overline{\mathrm{Y}}}{\beta_{2} \overline{\mathrm{X}}+\rho}$ & $\frac{(1-f)}{n} \frac{S_{x}^{2}}{\bar{Y}} R_{13}^{2}$ & $\frac{(1-f)}{n}\left(R_{13}^{2} S_{x}^{2}+S_{y}^{2}\left(1-\rho^{2}\right)\right)$ \\
\hline $\begin{array}{l}\hat{\bar{Y}}_{14}=\frac{\bar{y}+b(\bar{X}-\bar{x})}{\left(\rho \bar{x}+\beta_{2}\right)}\left(\rho \bar{X}+\beta_{2}\right) \\
\text { Kadilar and Cingi }{ }^{5}\end{array}$ & $\mathrm{R}_{14}=\frac{\rho \overline{\mathrm{Y}}}{\rho \overline{\mathrm{X}}+\beta_{2}}$ & $\frac{(1-\mathrm{f})}{\mathrm{n}} \frac{\mathrm{S}_{\mathrm{x}}^{2}}{\overline{\mathrm{Y}}} \mathrm{R}_{14}^{2}$ & $\frac{(1-f)}{n}\left(R_{14}^{2} S_{x}^{2}+S_{y}^{2}\left(1-\rho^{2}\right)\right)$ \\
\hline
\end{tabular}


Published by Atlantis Press

Copyright: the authors 336 
For a more detailed discussion on the ratio estimator and its modifications one may refer to Upadhyaya and Singh ${ }^{1}$, Singh $^{2}$, Yan and Tian ${ }^{3}$, Kadilar and Cingi ${ }^{4}$ and 5 , Cochran ${ }^{6}$, Khoshnevisan et al. ${ }^{7}$, Koyuncu and Kadilar ${ }^{8}$, Murthy ${ }^{9}$, Prasad $^{10}$, Rao ${ }^{11}$, Singh and Chaudhary ${ }^{12}$, Singh and Tailor ${ }^{13}$ and ${ }^{14}$, Singh et al. ${ }^{15}$, Sisodia and Dwivedi ${ }^{16}$, Subramani and Kumarapandiyan $^{17}$ and 18 and Tailor and Sharma ${ }^{19}$. The modified ratio estimators given in Table 1 and Table 2 are biased but have smaller mean squared errors compared to the classical ratio estimator. The list of estimators given in Table 1 and Table 2 uses the linear combinations of the known values of the parameters like $\bar{X}, C_{x}, \beta_{1}, \beta_{2}, \rho$ and $M_{d}$. However, it seems, no attempt is made to use the linear combination of known values of the Correlation Coefficient and Median of the auxiliary variable to improve the ratio estimator. The points discussed above have motivated us to introduce modified ratio estimators using the linear combination of the known values of Correlation Coefficient and Median of the auxiliary variable. It is observed that the proposed estimators perform better than the existing modified ratio estimators listed in Table 1 and Table 2. The materials of this paper are arranged as follows: The proposed modified ratio estimators using the linear combination of the known values of the Correlation Coefficient and Median of the auxiliary variable are presented in section 2 where as the conditions in which the proposed estimators perform better than the existing modified ratio estimators are derived in section 3. The performances of the proposed modified ratio estimators and the existing modified ratio estimators are assessed for certain natural populations in section 4 and the conclusion is presented in section 5

\section{Proposed Modified Ratio Estimators}

In this section, we have suggested two modified ratio estimators using the linear combination of Correlation Coefficient and Median of the auxiliary variable. The proposed modified ratio estimators for estimating the population mean $\bar{Y}$ together with the first degree of approximation, the biases and mean squared errors are given below:

$$
\begin{aligned}
& \hat{\bar{Y}}_{p_{1}}=\bar{y}\left[\frac{\rho \bar{X}+M_{d}}{\rho \bar{x}+M_{d}}\right] \\
& B\left(\hat{\bar{Y}}_{p_{1}}\right)=\frac{(1-f)}{n} \bar{Y}\left(\theta_{p_{1}}^{2} C_{x}^{2}-\rho \theta_{p_{1}} C_{x} C_{y}\right) \\
& \operatorname{MSE}\left(\hat{\bar{Y}}_{p_{1}}\right)=\frac{(1-f)}{n} \bar{Y}^{2}\left(C_{y}^{2}+\theta_{p_{1}}^{2} C_{x}^{2}-2 \rho \theta_{p_{1}} C_{x} C_{y}\right) \\
& \text { where } \theta_{p_{1}}=\frac{\rho \bar{X}}{\rho \bar{X}+M_{d}} \\
& \hat{\bar{Y}}_{p_{2}}=\frac{\bar{y}+b(\bar{X}-\bar{x})}{\left(\rho \bar{x}+M{ }_{d}\right)}\left(\rho \bar{X}+M_{d}\right) \\
& B\left(\hat{\bar{Y}}_{p_{2}}\right)=\frac{(1-f)}{n} \frac{S_{x}^{2}}{\bar{Y}} R_{p_{2}}^{2} \\
& \text { MSE }\left(\hat{\bar{Y}}_{p_{2}}\right)=\frac{(1-f)}{n}\left(R_{p_{2}}^{2} S_{x}^{2}+S_{y}^{2}\left(1-\rho^{2}\right)\right) \\
& \text { where } R_{p_{2}}=\frac{\rho \bar{Y}}{\rho \bar{X}+M_{d}}
\end{aligned}
$$

\section{Efficiency Comparison}

For want of space; for the sake of convenience to the readers and for the ease of comparisons, the modified ratio estimators given in Table 1, Table 2 are represented into two classes as given below: 
Class 1:The biases, the mean squared errors and the constants of the modified ratio type estimators $\hat{\bar{Y}}_{1}$ to $\hat{\bar{Y}}_{7}$ listed in the Table 1 are represented in a single class (say, Class 1), which will be very much useful for comparing with that of proposed modified ratio estimator $\hat{\bar{Y}}_{\mathrm{p}_{1}}$ and are given below:

$\mathrm{B}\left(\hat{\overline{\mathrm{Y}}}_{\mathrm{i}}\right)=\frac{(1-\mathrm{f})}{\mathrm{n}} \overline{\mathrm{Y}}\left(\theta_{\mathrm{i}}^{2} \mathrm{C}_{\mathrm{x}}^{2}-\rho \theta_{\mathrm{i}} \mathrm{C}_{\mathrm{x}} \mathrm{C}_{\mathrm{y}}\right)$

$\operatorname{MSE}\left(\hat{\overline{\mathrm{Y}}}_{\mathrm{i}}\right)=\frac{(1-\mathrm{f})}{\mathrm{n}} \overline{\mathrm{Y}}^{2}\left(\mathrm{C}_{\mathrm{y}}^{2}+\theta_{\mathrm{i}}^{2} \mathrm{C}_{\mathrm{x}}^{2}-2 \rho \theta_{\mathrm{i}} \mathrm{C}_{\mathrm{x}} \mathrm{C}_{\mathrm{y}}\right) i=1,2, \ldots, 6$

where $\theta_{1}=\frac{C_{x} \bar{X}}{C_{x} \bar{X}+\beta_{2}}, \theta_{2}=\frac{\beta_{2} \bar{X}}{\beta_{2} \bar{X}+C_{x}}, \theta_{3}=\frac{\beta_{1} \bar{X}}{\beta_{1} \bar{X}+S_{x}}, \theta_{4}=\frac{\beta_{2} \bar{X}}{\beta_{2} \bar{X}+S_{x}}, \theta_{5}=\frac{\beta_{2} \bar{X}}{\beta_{2} \bar{X}+\beta_{1}}, \theta_{6}=\frac{\beta_{1} \bar{X}}{\beta_{1} \bar{X}+\beta_{2}}$ and

$\theta_{7}=\frac{C_{x} \bar{X}}{C_{x} \bar{X}+\beta_{1}}$

Class 2:The biases, the mean squared errors and the constants of the remaining 7 modified ratio estimators $\hat{\bar{Y}}_{8}$ to $\hat{\bar{Y}}_{14}$ listed in the Table 2 are represented in a single class (say, Class 2), which will be very much useful for comparing with that of proposed modified ratio estimator $\hat{\overline{\mathrm{Y}}}_{\mathrm{p}_{2}}$ and are given below:

$B\left(\hat{\bar{Y}}_{i}\right)=\frac{(1-f)}{n} \frac{S_{x}^{2}}{\bar{Y}} R_{i}^{2}$

$\operatorname{MSE}\left(\hat{\bar{Y}}_{\mathrm{i}}\right)=\frac{(1-\mathrm{f})}{\mathrm{n}}\left(\mathrm{R}_{\mathrm{i}}^{2} \mathrm{~S}_{\mathrm{x}}^{2}+\mathrm{S}_{\mathrm{y}}^{2}\left(1-\rho^{2}\right)\right) ; \mathrm{i}=8,9, \ldots, 14$

where $R_{8}=\frac{\beta_{2} \bar{Y}}{\beta_{2} \bar{X}+C_{x}}, R_{9}=\frac{C_{x} \bar{Y}}{C_{x} \bar{X}+\beta_{2}} R_{10}=\frac{\beta_{1} \bar{Y}}{\beta_{1} \bar{X}+\beta_{2}} R_{11}=\frac{C_{x} \bar{Y}}{C_{x} \bar{X}+\rho}, R_{12}=\frac{\rho \bar{Y}}{\rho \bar{X}+C_{x}}, R_{13}=\frac{\beta_{2} \bar{Y}}{\beta_{2} \bar{X}+\rho}$ and

$R_{14}=\frac{\rho \bar{Y}}{\rho \bar{X}+\beta_{2}}$

As derived earlier in section 2, the biases, the mean squared errors and the constants of the proposed modified ratio estimators are given below:

$$
\begin{aligned}
& \mathrm{B}\left(\hat{\mathrm{Y}}_{\mathrm{p}_{1}}\right)=\frac{(1-\mathrm{f})}{\mathrm{n}} \overline{\mathrm{Y}}\left(\theta_{\mathrm{p}_{1}}^{2} \mathrm{C}_{\mathrm{x}}^{2}-\rho \theta_{\mathrm{p}_{1}} \mathrm{C}_{\mathrm{x}} \mathrm{C}_{\mathrm{y}}\right) \\
& \operatorname{MSE}\left(\hat{\overline{\mathrm{Y}}}_{\mathrm{p}_{1}}\right)=\frac{(1-\mathrm{f})}{\mathrm{n}} \overline{\mathrm{Y}}^{2}\left(\mathrm{C}_{\mathrm{y}}^{2}+\theta_{\mathrm{p}_{1}}^{2} \mathrm{C}_{\mathrm{x}}^{2}-2 \rho \theta_{\mathrm{p}_{1}} \mathrm{C}_{\mathrm{x}} \mathrm{C}_{\mathrm{y}}\right)
\end{aligned}
$$

where $\theta_{\mathrm{p}_{1}}=\frac{\rho \overline{\mathrm{X}}}{\rho \overline{\mathrm{X}}+\mathrm{M}_{\mathrm{d}}}$

$B\left(\hat{\bar{Y}}_{\mathrm{p}_{2}}\right)=\frac{(1-\mathrm{f})}{\mathrm{n}} \frac{S_{\mathrm{x}}^{2}}{\overline{\mathrm{Y}}} \mathrm{R}_{\mathrm{p}_{2}}^{2}$

$\operatorname{MSE}\left(\hat{\bar{Y}}_{\mathrm{p}_{2}}\right)=\frac{(1-\mathrm{f})}{\mathrm{n}}\left(\mathrm{R}_{\mathrm{p}_{2}}^{2} \mathrm{~S}_{\mathrm{x}}^{2}+\mathrm{S}_{\mathrm{y}}^{2}\left(1-\rho^{2}\right)\right)$

where $R_{p_{2}}=\frac{\rho \bar{Y}}{\rho \bar{X}+M_{d}}$ 
From the expressions given in (4) and (6) we have derived the conditions for which the proposed estimator $\hat{\bar{Y}}_{p_{1}}$ is more efficient than the existing modified ratio estimators given in Class $1, \hat{\bar{Y}}_{i} ; i=1,2,3, \ldots, 7$ and are given below:

$$
\operatorname{MSE}\left(\hat{\bar{Y}}_{\mathrm{p}_{1}}\right) \leq \operatorname{MSE}\left(\hat{\bar{Y}}_{\mathrm{i}}\right) \text { if } \rho \leq \frac{\left(\theta_{\mathrm{p}_{1}}+\theta_{\mathrm{i}}\right)}{2} \frac{\mathrm{C}_{\mathrm{x}}}{\mathrm{C}_{\mathrm{y}}} ; \mathrm{i}=1,2, \ldots, 7
$$

From the expressions given in (5) and (7) we have derived the conditions for which the proposed estimator $\hat{\bar{Y}}_{p_{2}}$ is more efficient than the existing modified ratio estimators given in Class $2, \hat{\bar{Y}}_{i} ; i=8,9,10, \ldots ., 14$ and are given below:

$$
\operatorname{MSE}\left(\hat{\overline{\mathrm{Y}}}_{\mathrm{p}_{2}}\right) \leq \operatorname{MSE}\left(\hat{\overline{\mathrm{Y}}}_{\mathrm{i}}\right) \text { if } \mathrm{R}_{\mathrm{p}_{2}}<\mathrm{R}_{\mathrm{i}} ; \mathrm{i}=8,9,10, \ldots, 14
$$

\section{Empirical Study}

The performances of the proposed modified ratio estimators listed are assessed with that of existing modified ratio estimators listed in Table 1 and Table 2 for certain natural populations. In this connection, we have considered three natural populations for the assessment of the performances of the proposed modified ratio estimators with that of existing modified ratio estimators. The population 1 is taken from Singh and Chaudhary ${ }^{2}$ given in page 141, the population 2 is taken from Cochran ${ }^{6}$ given in page 152 and population 3 is the closing price of the industry ACC in the National Stock Exchange from 2, January 2012 to 27 , February $2012^{20}$. The population parameters and the constants computed from the above populations are given below:

Table 3: Parameters and Constants of the Populations

\begin{tabular}{|c|r|r|r|}
\hline Parameters & Population 1 & Population 2 & Population 3 \\
\hline $\mathrm{N}$ & 22 & 49 & 40 \\
\hline $\mathrm{n}$ & 5 & 20 & 20 \\
\hline$\overline{\mathrm{Y}}$ & 22.6201 & 116.1633 & 5141.5363 \\
\hline$\overline{\mathrm{X}}$ & 1467.5455 & 98.6735 & 1221.6463 \\
\hline$\rho$ & 0.9021 & 0.6904 & 0.9244 \\
\hline $\mathrm{S}_{\mathrm{x}}$ & 33.0469 & 98.8286 & 256.1464 \\
\hline $\mathrm{C}_{\mathrm{y}}$ & 1.4601 & 0.8508 & 0.0557 \\
\hline $\mathrm{S}_{\mathrm{x}}$ & 2562.1449 & 102.9709 & 102.5494 \\
\hline $\mathrm{C}_{\mathrm{x}}$ & 1.7459 & 1.0436 & 0.0839 \\
\hline$\beta_{2}$ & 13.3693 & 5.9878 & -1.5154 \\
\hline$\beta_{1}$ & 3.3914 & 2.4224 & 0.3761 \\
\hline $\mathrm{M}_{\mathrm{d}}$ & 534.5000 & 64.0000 & 1184.2250 \\
\hline
\end{tabular}

The constants of the existing and proposed modified ratio estimators for the above populations are given in the Table 4 and Table 5: 
Table 4: The constants of the (Class 1) existing and proposed modified ratio estimators

\begin{tabular}{|c|c|c|c|}
\hline \multirow{2}{*}{ Estimator } & \multicolumn{3}{|c|}{ Constants $\theta_{i}$} \\
\cline { 2 - 4 } & Population 1 & Population 2 & Population 3 \\
\hline$\hat{\overline{\mathrm{Y}}}_{1}$ & 0.9948 & 0.9450 & 1.0150 \\
\hline$\hat{\overline{\mathrm{Y}}}_{2}$ & 0.9999 & 0.9982 & 1.0000 \\
\hline$\hat{\overline{\mathrm{Y}}}_{3}$ & 0.6602 & 0.6989 & 0.8175 \\
\hline$\hat{\overline{\mathrm{Y}}}_{4}$ & 0.8845 & 0.8516 & 1.0586 \\
\hline$\hat{\overline{\mathrm{Y}}}_{5}$ & 0.9998 & 0.9959 & 1.0002 \\
\hline$\hat{\overline{\mathrm{Y}}}_{6}$ & 0.9973 & 0.9756 & 1.0033 \\
\hline$\hat{\bar{Y}}_{7}$ & 0.9987 & 0.9770 & 0.9963 \\
\hline$\hat{\overline{\mathrm{Y}}}_{\mathrm{p}_{1}}$ & $0.7124 *$ & $0.5156^{*}$ & $0.4881^{*}$ \\
\hline
\end{tabular}

Table 5: The constants of the (Class 2) existing and proposed modified ratio estimators

\begin{tabular}{|c|c|c|c|}
\hline \multirow{2}{*}{ Estimator } & \multicolumn{3}{|c|}{ Constants $\mathrm{R}_{\mathrm{i}}$} \\
\cline { 2 - 4 } & Population 1 & Population 2 & Population 3 \\
\hline$\hat{\overline{\mathrm{Y}}}_{8}$ & 0.0154 & 1.1752 & 4.2089 \\
\hline$\hat{\overline{\mathrm{Y}}}_{9}$ & 0.0153 & 1.1126 & 4.2718 \\
\hline$\hat{\bar{Y}}_{10}$ & 0.0154 & 1.1485 & 4.2226 \\
\hline$\hat{\bar{Y}}_{11}$ & 0.0154 & 1.1694 & 4.1711 \\
\hline$\hat{\overline{\mathrm{Y}}}_{12}$ & 0.0154 & 1.1595 & 4.2084 \\
\hline$\hat{\bar{Y}}_{13}$ & 0.0154 & 1.1759 & 4.2108 \\
\hline$\hat{\bar{Y}}_{14}$ & 0.0153 & 1.0821 & 4.2143 \\
\hline$\hat{\bar{Y}}_{\mathrm{p}_{2}}$ & $0.0110^{*}$ & $0.6070^{*}$ & $2.0544 *$ \\
\hline
\end{tabular}

The biases of the existing and proposed modified ratio estimators for the above populations are given in the Table 6 and Table 7:

Table 6: The biases of the (Class 1) existing and proposed modified ratio estimators

\begin{tabular}{|c|c|c|c|}
\hline \multirow{2}{*}{ Estimator } & \multicolumn{3}{|c|}{ Bias B(.) } \\
\cline { 2 - 4 } & Population 1 & Population 2 & Population 3 \\
\hline$\hat{\bar{Y}}_{1}$ & 2.5432 & 1.3519 & 0.3697 \\
\hline$\hat{\bar{Y}}_{2}$ & 2.6106 & 1.6268 & 0.3507 \\
\hline$\hat{\bar{Y}}_{3}$ & 0.6665 & 0.3559 & 0.1515 \\
\hline$\hat{\bar{Y}}_{4}$ & 1.2215 & 0.9203 & 0.4274 \\
\hline$\hat{\bar{Y}}_{5}$ & 2.6095 & 1.6144 & 0.3509 \\
\hline$\hat{\bar{Y}}_{6}$ & 2.5763 & 1.5070 & 0.3548 \\
\hline$\hat{\bar{Y}}_{7}$ & 2.5943 & 1.5146 & 0.3460 \\
\hline$\hat{\bar{Y}}_{p_{1}}$ & $0.3226^{*}$ & $0.0913 *$ & $0.0552 *$ \\
\hline
\end{tabular}


Table 7: The biases of the (Class 2) existing and proposed modified ratio estimators

\begin{tabular}{|c|c|c|c|}
\hline \multirow{2}{*}{ Estimator } & \multicolumn{3}{|c|}{ Bias B(.) } \\
\cline { 2 - 4 } & Population 1 & Population 2 & Population 3 \\
\hline$\hat{\bar{Y}}_{8}$ & 10.6540 & 3.7302 & 0.9058 \\
\hline$\hat{\bar{Y}}_{9}$ & 10.5456 & 3.3433 & 0.9331 \\
\hline$\hat{\bar{Y}}_{10}$ & 10.5989 & 3.5627 & 0.9118 \\
\hline$\hat{\bar{Y}}_{11}$ & 10.6484 & 3.6937 & 0.8896 \\
\hline$\hat{\bar{Y}}_{12}$ & 10.6279 & 3.6313 & 0.9056 \\
\hline$\hat{\bar{Y}}_{13}$ & 10.6549 & 3.7347 & 0.9067 \\
\hline$\hat{\bar{Y}}_{14}$ & 10.4439 & 3.1630 & 0.9082 \\
\hline$\hat{\bar{Y}}_{p_{2}}$ & $5.4079 *$ & $0.9953^{*}$ & $0.2158^{*}$ \\
\hline
\end{tabular}

The mean squared errors of the existing and proposed modified ratio estimators for the above populations are given in the Table 8 and Table 9:

Table 8: The mean squared errors of the (Class 1) existing and proposed modified ratio estimators

\begin{tabular}{|c|c|c|c|}
\hline \multirow{2}{*}{ Estimator } & \multicolumn{3}{|c|}{ Mean Squared Error MSE(.) } \\
\cline { 2 - 4 } & Population 1 & Population 2 & Population 3 \\
\hline$\hat{\bar{Y}}_{1}$ & 45.2894 & 214.7486 & 1050.6525 \\
\hline$\hat{\bar{Y}}_{2}$ & 45.8857 & 233.6573 & 995.6899 \\
\hline$\hat{\bar{Y}}_{3}$ & 33.5787 & 159.2888 & 492.6945 \\
\hline$\hat{\bar{Y}}_{4}$ & 35.4638 & 187.4850 & 1222.9729 \\
\hline$\hat{\bar{Y}}_{5}$ & 45.8758 & 232.7813 & 996.2592 \\
\hline$\hat{\bar{Y}}_{6}$ & 45.5814 & 225.2956 & 1007.5083 \\
\hline$\hat{\bar{Y}}_{7}$ & 45.7405 & 225.8185 & 982.4136 \\
\hline$\hat{\bar{Y}}_{p_{1}}$ & $31.8505^{*}$ & $152.2157 *$ & $370.1528 *$ \\
\hline
\end{tabular}

Table 9: The mean squared errors of the (Class 2) existing and proposed modified ratio estimators

\begin{tabular}{|c|c|c|c|}
\hline \multirow{2}{*}{ Estimator } & \multicolumn{3}{|c|}{ Mean Squared Error MSE(.) } \\
\cline { 2 - 4 } & Population 1 & Population 2 & Population 3 \\
\hline$\hat{\bar{Y}}_{8}$ & 272.4185 & 584.5606 & 4955.0419 \\
\hline$\hat{\bar{Y}}_{9}$ & 269.9654 & 539.6120 & 5095.3661 \\
\hline$\hat{\bar{Y}}_{10}$ & 271.1716 & 565.0981 & 4985.4911 \\
\hline$\hat{\bar{Y}}_{11}$ & 272.2918 & 580.3192 & 4871.7809 \\
\hline$\hat{\bar{Y}}_{12}$ & 271.8270 & 573.0710 & 4953.9273 \\
\hline$\hat{\bar{Y}}_{13}$ & 272.4393 & 585.0781 & 4959.2739 \\
\hline$\hat{\bar{Y}}_{14}$ & 267.6660 & 518.6688 & 4967.1427 \\
\hline$\hat{\bar{Y}}_{p_{2}}$ & $153.7472 *$ & $266.8558^{*}$ & $1407.3186^{*}$ \\
\hline
\end{tabular}


From the values of Table 6 and Table 7, it is observed that the bias of the proposed modified ratio estimator $\hat{\bar{Y}}_{p_{1}}$ is less than the biases of the existing modified ratio estimators $\hat{\bar{Y}}_{i} ; i=1,2,3, \ldots, 7$ given in Class 1 and the bias of the proposed modified ratio estimator $\hat{\bar{Y}}_{\mathrm{p}_{2}}$ is less than the biases of the existing modified ratio estimators $\hat{\bar{Y}}_{\mathrm{i}} ; \mathrm{i}=8,9,10, \ldots, 14$ given in Class 2. Similarly from the values of Table 8 and Table 9, it is observed that the mean squared error of the proposed modified ratio estimator $\hat{\bar{Y}}_{\mathrm{p}_{1}}$ is less than the mean squared errors of the existing modified ratio estimators $\hat{\bar{Y}}_{i} ; i=1,2,3, \ldots, 7$ given in Class 1 and the mean squared error of the proposed modified ratio estimator $\hat{\bar{Y}}_{p_{2}}$ is less than the mean squared errors of the existing modified ratio $\hat{\bar{Y}}_{i} ; i=8,9,10, \ldots, 14$ given in Class 2 .

\section{Conclusion}

In this paper we have proposed two modified ratio estimators using linear combination of Correlation Coefficient and Median of the auxiliary variable. The biases and mean squared errors of the proposed estimators are obtained and compared with that of existing modified ratio estimators. Further we have derived the conditions for which the proposed estimators are more efficient than the existing modified ratio estimators. We have also assessed the performances of the proposed estimators for some known populations. It is observed that the biases and mean squared errors of the proposed estimators are less than the biases and mean squared errors of the existing modified ratio estimators for certain known populations. Hence we strongly recommend that the proposed modified estimators may be preferred over the existing modified ratio estimators for the use of practical applications.

\section{Acknowledgement}

The authors are thankful to the editor and the reviewers for their constructive comments, which have improved the presentation of the paper. The authors also wish to record their gratitude and thanks for the financial assistance received through UGC-Major Research Project and Department of Science and Technology-INSPIRE fellowship, New Delhi.

\section{References}

1. L.N. Upadhyaya and H.P. Singh, Use of transformed auxiliary variable in estimating the finite population mean, Biometrical Journal 41(5) (1999) 627-636

2. G.N. Singh, On the improvement of product method of estimation in sample surveys, Journal of the Indian Society of Agricultural Statistics 56(3) (2003) 267-265

3. Z. Yan and B. Tian, Ratio method to the mean estimation using coefficient of skewness of auxiliary variable, ICICA, Part II, CCIS 106 (2010) 103-110

4. C. Kadilar and H. Cingi, Ratio estimators in simple random sampling, Applied Mathematics and Computation 151 (2004) 893-902

5. C. Kadilar and H. Cingi, An Improvement in estimating the population mean by using the correlation co-efficient, Hacettepe Journal of Mathematics and Statistics 35 (1) (2006) 103-109

6. W.G. Cochran, Sampling Techniques, Third Edition, (Wiley Eastern Limited, 1977)

7. M. Khoshnevisan, R. Singh, P. Chauhan, N. Sawan and F. Smarandache, A general family of estimators for estimating mean using known value of population parameters, Far East Journal of Theoretical Statistics 22 (2007) 181-191

8. N. Koyuncu and C. Kadilar, Efficient estimators for the population mean, Hacettepe Journal of Mathematics and Statistics 38(2), (2009) 217-225

9. M.N. Murthy, Sampling theory and methods, (Statistical Publishing Society, Calcutta, India, 1967)

10. B. Prasad, Some improved ratio type estimators of population mean and ratio in finite population sample surveys, Communications in Statistics: Theory and Methods 18 (1989) 379-392

11. T.J. Rao, On certain methods of improving ratio and regression estimators, Communications in Statistics: Theory and Methods 20 (10) (1991) 3325-3340

12. D. Singh and F.S. Chaudhary, Theory and analysis of sample survey designs, (New Age International Publisher, 1986)

13. H.P. Singh and R. Tailor, Use of known correlation co-efficient in estimating the finite population means, Statistics in Transition 6(4) (2003) 555-560 
14. H.P. Singh and R. Tailor, Estimation of finite population mean with known coefficient of variation of an auxiliary, STATISTICA anno LXV, n.3 (2005) 301-313

15. H.P. Singh, R. Tailor, R. Tailor and M.S. Kakran, An improved estimator of population mean using power transformation, Journal of the Indian Society of Agricultural Statistics 58(2) (2004) 223-230

16. B.V.S. Sisodia and V.K. Dwivedi, A modified ratio estimator using co-efficient of variation of auxiliary variable, Journal of the Indian Society of Agricultural Statistics 33(1) (1981)13-18

17. J. Subramani and G. Kumarapandiyan, Estimation of population mean using coefficient of variation and median of an auxiliary variable, International Journal of Probability and Statistics 1(4) (2012) 111-118

18. J. Subramani and G. Kumarapandiyan, A new modified ratio estimator for estimation of population mean when median of the auxiliary variable is known, Pakistan Journal of Statistics and Operation Research 9(2) (2013) 137-145

19. R. Tailor and B.K. Sharma, A modified ratio-cum-product estimator of finite population mean using known coefficient of variation and coefficient of kurtosis, Statistics in Transition-new series, 10(1) (2009) 15-24

20. www.nseindia.com/index.htm Historical Security-wise Price Volume Data- Data for ACC - EQ from 02-01-2012 to 27-02-2012 